\title{
A SIMPLE CONSTRUCTION OF ANALYTIC FUNCTIONS WITHOUT RADIAL LIMITS
}

\author{
DAVID G. CANTOR
}

The purpose of this note is to give a simple construction of a class of functions, analytic in the unit disc, having radial limits nowhere. We first prove, as a lemma, an easy Tauberian theorem, and use it to establish our result.

Let $n_{1}<n_{2}<n_{3}<\cdots$ be an increasing sequence of positive integers satisfying

$$
\frac{r_{k}^{n_{k+1}}}{1-r_{k}} \leqq \frac{1}{2^{k}}, \quad k=1,2,3, \cdots,
$$

where $r_{k}$ is defined as the positive solution of $r_{k}^{n_{k}}=1-2^{-k}$. Such a sequence can easily be constructed by induction. Clearly $\lim _{k \rightarrow \infty} r_{k}=1$; and if $k \leqq j$, then $1-r_{j}^{n_{k}} \leqq 1 / 2^{j}$.

Lemma. Suppose $a_{i}$ is a bounded sequence of complex numbers satisfying

$$
\lim _{r \rightarrow 1-} \sum_{k=1}^{\infty} a_{k} r^{n_{k}}=s
$$

Then

$$
\sum_{k=1}^{\infty} a_{k}=s .
$$

Proof. Let $A$ be a bound for the $a_{k}$. Then

$$
\begin{aligned}
\left|\sum_{k=1}^{j} a_{i}-\sum_{k=1}^{\infty} a_{k} r_{j}^{n_{k}}\right| & \leqq\left|\sum_{k=1}^{j} a_{k}\left(1-r_{j}^{n_{k}}\right)\right|+\left|\sum_{k=j+1}^{\infty} a_{k} r_{j}^{n_{k}}\right| \\
& \leqq A j / 2^{j}+A / 2^{j} .
\end{aligned}
$$

TheOREM 1. Suppose $\left\{b_{k}\right\}$ is a bounded sequence of complex numbers not satisfying $\lim _{k \rightarrow \infty} b_{k}=0$. Then, if $n_{k}$ satisfies (1), $f(z)=\sum_{k=1}^{\infty} b_{k} z^{n_{k}}$ is analytic in the unit disc, and $f(z)$ has radial limits nowhere.

Proof. Clearly, $f(z)$ is analytic in $|z|<1$. Suppose $f(z)$ has a radial limit at $z=e^{i \theta}$, i.e.,

Received by the editors November 2, 1962 and, in revised form, February 12, 1963. 


$$
\lim _{r \rightarrow 1-} \sum_{j=1}^{\infty} b_{j} e^{i n_{j} \theta_{r} n_{j}}
$$

exists. By the Lemma,

$$
\sum_{j=1}^{\infty} b_{j} e^{i n j \theta}
$$

exists; hence $\lim _{j \rightarrow \infty} b_{j}=0$, contradicting the hypothesis.

The following theorem was suggested by a question of M. H. Heins.

TheORem 2. Let $\phi(r)$ be a continuous, strictly positive function, defined for $0 \leqq r<1$, satisfying $\lim _{r \rightarrow 1-} \phi(r)=\infty$; let $\left\{b_{k}\right\}$ be a bounded sequence of complex numbers not satisfying $\lim _{k \rightarrow \infty} b_{k}=0$. Then, there exists an increasing sequence of integers $\left\{n_{k}\right\}$, such that $f(z)=\sum_{k=1}^{\infty} b_{k} z^{n_{k}}$ is analytic in the unit disc, has radial limits nowhere, and $\max _{|z|=r}|f(z)|$ $\leqq \phi(r)$.

Proof. Choose the integer $n_{1}$ so large that

$$
\left|b_{1}\right| r^{n_{1}} \leqq 2^{-1} \phi(r)
$$

for $0 \leqq r<1$. Now, inductively, given $n_{k}$, choose $n_{k+1}$ so large that (1) is satisfied, and that

$$
\left|b_{k+1}\right| r^{n_{k+1}} \leqq 2^{-k-1} \phi(r),
$$

for $0 \leqq r<1$. Then, by Theorem 1 ,

$$
f(z)=\sum_{k=1}^{\infty} b_{k} z^{n k}
$$

is analytic in the unit disc and has radial limits nowhere. By (2) and (3),

$$
\max _{|z|=r}|f(z)| \leqq \sum_{k=1}^{\infty}\left|b_{k}\right| r^{n_{k}} \leqq \sum_{k=1}^{\infty} 2^{-k} \phi(r)=\phi(r) .
$$

UNIVERSITY OF WASHINGTON 